\title{
A comprehensive review of remimazolam for sedation
}

\author{
Nazir Noor, MD ${ }^{1}$, Rhorer Legendre, BS ${ }^{2}$, Alexandra Cloutet ${ }^{2}$, Ahish Chitneni ${ }^{3}$, Giustino Varrassi, MD, PhD, FIPP ${ }^{4}$, Alan $^{2}$ \\ D. Kaye, MD, PhD ${ }^{2}$ \\ ${ }^{1}$ Department of Anesthesiology, Mount Sinai Medical Center, Miami Beach, FL, ${ }^{2}$ Louisiana State University Health Sciences Center School of \\ Medicine, Shreveport, LA, ${ }^{3}$ AT Still University School of Osteopathic Medicine, Mesa, AZ, 4 Paolo Procacci Foundation, Roma, Itlay \\ Keywords: sedation, benzodiazepine, remimazolam \\ https://doi.org/10.52965/001c.24514
}

\section{Health Psychology Research}

Vol. 9, Issue 1, 2021

Benzodiazepines are one of the most commonly used medications in the field of anesthesia. They offer excellent anxiolytic and amnestic properties ideal for the perioperative period when patient anxiety is understandably heightened. Remimazolam has presented a favorable alternative to some of the common intravenous anesthetic agents used given its fast onset of action, high safety profile, and reasonably short duration of action.

The drugs within the four classes of benzodiazepines, 2-keto-benzodiazepines, 3-hydroxy-benzodiazepines, triazolo-benzodiazepines, and 7-nitro-benzodiazepines provide varying degrees of anxiolysis, sedation, and amnesia. This is provided by the benzodiazepine molecule binding and causing a conformational change to the chloride ion channel to cause hyperpolarization and thus inhibition of the central nervous system. Each type of benzodiazepine has a preferred role within the realm of medicine. For instance, diazepam is used for the treatment of seizures and anxiety. Midazolam's anxiolytic and anterograde amnestic properties are taking advantage of during the perioperative period. Lorazepam is beneficial for anxiety and status epilepticus. Remimazolam, currently in phase II and III clinical trials, has demonstrated a very short during of action and low context-sensitive half-time, allowing for its rapid removal even during a prolonged infusion. Much of its properties may be credited to being a soft drug, meaning it is a metabolically active drug that is rapidly inactivated in the body. This provides anesthesiologists and other practitioners administering it with a more predictable sedative. These properties have the potential to push it towards becoming the drug of choice for premedication during the perioperative period and sedation in the ICU. Furthermore, remimazolam does not seem to rely on any specific organ to be metabolized. The drug's ester moiety makes it a substrate for non-specific tissue esterase enzymes, meaning its metabolism and elimination are not impaired in patients with hepatic and/or renal disease. Its addictive potential closely resembles that of its parent compound, midazolam. Reports of its adverse reactions include headache and somnolence after an involuntary movement during infusion.

Benzodiazepines are a great adjunct to anesthetic care. Remimazolam's safety profile, pharmacokinetics, pharmacodynamics, and potential practical use make it quite favorable in this regard. It has the potential to equip anesthesiologists and other medical practitioners with a more predictable medication that has a good safety profile. However, further large clinical trials will provide us with a better understanding of the advantages and disadvantages of remimazolam.

\footnotetext{
a Corresponding Author:

Mount Sinai Medical Center

Department of Anesthesiology

4300 Alton Road

Miami Beach, FL 33130,

Phone: (925)-628-6259,

Email: naz9noor@gmail.com
} 


\section{INTRODUCTION}

Remimazolam is a benzodiazepine drug that is a recent innovation in the field of anesthesia. ${ }^{1}$ Benzodiazepines are a class of drugs that directly interact with gamma-aminobutyric acid (GABA) and modifiable chloride channels through polysynaptic pathway inhibition. ${ }^{2}$ In anesthesia, the anxiolytic properties of benzodiazepines make them useful as sedatives, which are loosely defined as the middle ground between naturally relaxed, and an unconscious state as one would be under general anesthesia. ${ }^{3}$ In terms of intravenous hypnotic agents, propofol has long been thought of as the "ideal agent" since the first administration of its current formulation (Diprivan) and its subsequent debut into clinical usage in 1986. However, scientists and clinicians continue to question how ideal propofol is due to certain risks. For example, its requirement for formulation in a lipid solution introduces the risk of bacterial contamination from open air. There have also been reports of pain on intravenous injection, aside from its most wellknown risk of cardiorespiratory depression. ${ }^{4,5}$ Midazolam is also not an optimal anesthesia drug, as although its effects of cardiorespiratory depression are less, it comes with the risk of excessive sedation. It can accumulate in situations such as renal impairment and prolonged infusion. Individual variability in cytochrome P450-3A4 activity can cause the drug's onset and duration to fluctuate unpredictably. Propofol has a fast onset of 9-51 seconds and recovery of 10 minutes but comes with a considerably unattractive safety profile; midazolam is safer and is the shortest acting benzodiazepine currently available on the US market but has an onset of 3-5 minutes and a recovery period of 2 hours. Remimazolam displays a fast onset of action, good safety profile, and short recovery time in comparison to intravenous (IV) sedatives commonly used in anesthesia, such as propofol and midazolam, and it is, therefore, a promising combination of the advantages of Propofol and midazolam. 6,7

\section{COMMON USE OF BENZODIAZEPINES}

Benzodiazepines primarily exert their effects in the amygdala and reticular activating system via direct interaction with GABA and modifiable chloride channels to cause polysynaptic pathway inhibition. ${ }^{2}$ GABA-A, one of three GABA receptor subtypes, is the site of action for benzodiazepines. ${ }^{8}$ This receptor has five subunits and is a ligand-gated, chloride-selective ion channel that forms a pocket between its two alpha and two gamma subunits. Benzodiazepines act as positive allosteric modulators where they bind at this pocket. ${ }^{2}$ Under normal circumstances, two GABA molecules bind the GABA-A receptor, whereas one benzodiazepine molecule occupies the entire binding site. ${ }^{9}$ This induces a conformational change in the chloride channel, causing it to hyperpolarize, leading to the desired effect of inhibiting the central nervous system. ${ }^{10}$

The most common use of benzodiazepines is for sedation, as well as for adjuvants to general anesthetics. ${ }^{2}$ Due to their ability to cause various responses in different patients, reduced potency, slow onset, and long-lasting residual ef- fects, benzodiazepines are not used as primary induction agents. ${ }^{11}$ When used as IV induction agents or sedatives, these drugs have advantages, such as water solubility, anxiolytic effects, and amnesia, as seen with midazolam. On the other hand, benzodiazepines such as diazepam are used primarily to manage conditions such as seizures and anxiety and are not used in perioperative settings due to their slow offset and ability to be a venous irritant. ${ }^{2,12}$ Benzodiazepines are classified by duration of action, elimination half-life, and/or chemical structure. Short-acting refers to a duration of fewer than 24 hours, while long-acting implies a duration of action greater than 24 hours. Factors that contribute to the duration of action include rate and degree of drug distribution, elimination profile following distribution, and the active metabolites produced upon drug breakdown. $^{2}$

Four groups of benzodiazepines are classified based on chemical structure and half-life: 2-keto-benzodiazepines, 3-hydroxy-benzodiazepines, triazolo-benzodiazepines, and 7-nitro-benzodiazepines. The 2-keto-benzodiazepines include diazepam, chlordiazepoxide, flurazepam, and many other early benzodiazepines. Diazepine is considered the prototype benzodiazepine drug, as it is low potency with a long half-life due to its extensive biotransformation before elimination from the body. It is hydroxylated at the three position to become temazepam, which is then demethylated to form oxazepam, both active drugs and members of the second class 3-hydroxy-benzodiazepines. Another member of this class is lorazepam, which is an exception to the low potency of the other 3-hydroxy-benzodiazepines. Oxazepam has the slowest onset of action and the lowest risk of dependency on the drugs in this class. The 3-hydroxy-benzodiazepines are good for detoxifying inpatients at risk for serious alcohol withdrawal or those with decreased hepatic function with the potential for liver failure.

Additionally, lorazepam can be administered intravenously or intramuscularly to provide a rapid onset of action when this effect is needed, such as for status epilepticus. Drugs in the triazolo-benzodiazepine class have an added ring structure, allowing them to be more ring soluble and have a shorter half-life and greater likelihood of withdrawal. Alprazolam, a member of this class, can do a crosstaper for patients administered clonazepam, as these two agents have similar benzodiazepine binding sites. In contrast, alprazolam has a considerably longer half-life that permits such a taper. The only member of the final class, 7-nitro-benzodiazepines, widely used in the United States, is clonazepam. It has a longer half-life, so once-daily administration is effective to reach target concentrations. ${ }^{13}$

An important clinical consideration for prescription use of benzodiazepines is associated with liability for abuse and dependence. Physicians should be aware that triazolo-benzodiazepines pose the greatest risk of abuse and dependence due to their high lipophilicity and rapid absorption. When it comes to pharmacological management of certain disorders, panic disorder and other chronic anxiety conditions are similar to epilepsy, may warrant benzodiazepines in their management. Despite their sedation effects, however, benzodiazepines do not make good sleeping aids due to the ability for circulating levels to persist after the individual awakens, impairing alertness and reaction time. ${ }^{13}$ 
In anesthesia, benzodiazepines are the standard drugs chosen for most procedural sedation regimens, regardless of the intended depth or route of administration. While they have little effect on cardiorespiratory function at doses recommended for at-home use for minimal sedation purposes, doses used in anesthesia for moderate or deep sedation reduce ventilation significantly, primarily by depressing hypoxemic drive and decreasing muscle tone in the upper airway. ${ }^{14}$

Several newer benzodiazepines could be clinically useful in the future. Among these is remimazolam, one of the most promising and currently in phase II and III clinical trials. ${ }^{2}$ This agent is known to be very short-acting as it is metabolized to an inactive carboxylic acid metabolite that is rapidly removed even during prolonged infusions. ${ }^{7}$ Its potential clinical uses include premedication for anesthesia, procedural sedation for endoscopic procedures, and sedation in ICU settings. ${ }^{2}$ Other new benzodiazepines include clonazolam, adinazolam, 3-hydroxyphenazapam, and 4-chlorodiazepam. Clonazolam is a derivative of clonazepam and alprazolam. It is a highly potent amnesic and sedative benzodiazepine. Its main uses are as a potent amnesic and sedative. ${ }^{2}$ When administered, it can have anxiolytic and muscle relaxant effects, making it appropriate in the initial treatment of generalized seizures. ${ }^{15,16}$ Adinazolam is a benzodiazepine derivative with anxiolytic, anticonvulsant, sedative, and antidepressant properties. It is used in the treatment of panic disorder, depression, and generalized anxiety disorder. ${ }^{2}$ Additionally, it can decrease catecholamine levels, which benefit patients with high levels such as those with pheochromocytomas, heart failure, or myocardial infarction. ${ }^{17}$ 3-hydroxyphenazapam is another new benzodiazepine, mainly used recreationally. However, its sedative properties are rarely used to treat epilepsy, alcohol withdrawal, and insomnia. It mimics lysergic acid diethylamide (LSD) and has similar effects as its parent compound, diazepam, with fewer myorelaxant effects. ${ }^{2}$ 4-chlorodiazepam is a derivative of diazepam currently in the research phase. Unlike the benzodiazepines previously discussed, it has no affinity for GABA-A receptors, giving this atypical drug effects. ${ }^{2}$ It inhibits oxidative stress and decreases cholesterol accumulation in the mitochondria during coronary reperfusion; thus, it has cardioprotective abilities. ${ }^{18}$ Additionally, it can decrease pro-inflammatory mast cell functions and can suppress LPS-induced TNF activity. ${ }^{19,20}$

\section{DRUG INFORMATION AND MECHANISM OF ACTION}

Remimazolam besilate (CNS 7056) was designed as a soft drug, metabolically active in its native form, and converted to an inactive compound rapidly. This is not to be confused with a prodrug, which is the converse (inactive metabolite predictably converted to an active metabolite). ${ }^{21}$ The advent of soft drugs is of particular importance to the field of anesthesia, as these new compounds give more control and predictability to anesthesiologists.

Remimazolam is a short-acting GABA-A receptor agonist. Its molecular formula is $\mathrm{C}_{21} \mathrm{H}_{19} \mathrm{BrN}_{4} \mathrm{O}_{2}$, with an aver- age mass of $439.305 \mathrm{Da}$. The structure of remimazolam is analogous to that of midazolam but with the addition of an ester moiety. It is water-soluble, and consequently, there is less pain at injection sites. ${ }^{22}$

\section{MECHANISM OF ACTION}

Remimazolam has a high affinity for the benzodiazepine (BDZ) site on GABA (A) brain receptors. It binds at the interface between alpha $(\alpha)$ and gamma $(r)$ subunits and acts to augment inhibitory activity in the central nervous system. This is achieved by increasing the intracellular concentration of chloride ions, leading to membrane hyperpolarization and subsequent inhibition of the neuron action potential conduction. Unlike some benzodiazepines, remimazolam does not display selectivity between the GABA-A receptor subtypes. However, it was observed to be slightly more potent at the $\alpha_{1}$-containing subtypes than midazolam. This is notable because $\alpha_{1}$-containing subtypes of GABA-A are thought to mediate sedation. ${ }^{23}$

\section{METABOLISM}

Unlike its parent compound (midazolam), remimazolam is not metabolized by cytochrome P-450 isozymes, nor does it inhibit cytochrome P-450 metabolism. ${ }^{6}$ The ester moiety of remimazolam is a substrate for non-specific tissue esterase enzymes, meaning it can be metabolized without the need of any particular organ. It has been suggested that this will make it a valuable drug for people with hepatic or renal compromise. $^{22}$

The metabolic profile of remimazolam was elucidated with the use of ultra-high-performance liquid chromatography/tandem mass spectrometry (UPLC/MS/MS). A total of five significant metabolites were characterized, of which three were phase I (oxidation, reduction, or hydrolysis of the parent drug), and two were phase II (conjugation by coupling the drug or its metabolites to another molecule) metabolites. ${ }^{24}$ Carboxylesterase enzymes are the most important players in remimazolam metabolism and can be found in the cytosol and the rough endoplasmic reticulum of tissues. ${ }^{25}$ Once acted upon by an esterase, the remimazolam is converted to its major inactive metabolite (CNS7054) that has a 400 -fold lower affinity for the GABA-A receptor. ${ }^{23}$

\section{ADDICTION}

When investigating a new benzodiazepine, it is always important to quantify the addictive capacity of the drug. Studies have confirmed equipotency when comparing remimazolam and midazolam with comparable levels of addictive potential. Both drugs have poor oral bioavailability and pose little risk of addiction when administered IV. ${ }^{26}$

\section{ADVERSE REACTIONS}

A few adverse events have been reported in conjunction with remimazolam administration. One study reported that the most common adverse events that occur after recovery post-infusion were headaches and drowsiness. ${ }^{27}$ Additionally, another study reported involuntary movement as the 
most notable adverse event during infusion. ${ }^{28}$

\section{REMIMAZOLAM USE}

The use of remimazolam will become especially beneficial, given that there is an ongoing problem in the United States because it has become standard for all patients undergoing GI endoscopic sedation to be administered propofol. This paradigm requires that an anesthesia provider be present, which is an unnecessary added cost to the patient and is unsustainable. $^{29}$ Alternatives to Propofol are especially sought out by gastroenterologists, as the use of propofol during procedures is legally binding due to the directive set out by the American Society of Anesthesiologists (ASA) "PropofolHealthcare providers must only use propofol with specialized training in airway management." ${ }^{30}$ Since most physicians beyond anesthesiologists and emergency physicians lack additional training in airway management, there is a need for an anesthesiologist to be present during procedures in which propofol is administered. 31

For procedures such as EGD (esophagogastroduodenoscopy) and ERCP (endoscopic retrograde cholangiopancreatography), remimazolam, when it becomes available, has the potential to be administered with oliceridine. Oliceridine is a mu-receptor agonist proposed to achieve adequate analgesia with the major benefit of reduced opioid-related adverse events, such as nausea and vomiting, compared to standard analgesics, like fentanyl. ${ }^{32}$ Additionally, oliceridine has a reduced potential to cause respiratory depression, with a minor drawback of an approximate five-minute increase in clinical onset of action. ${ }^{33}$

Although still under investigation in phase III trials, remimazolam has many properties that could deem it suitable for procedural sedation. Currently, trials are still ongoing to study the effects of these properties and how they compare to those of the frequently used propofol and midazolam. Remimazolam undergoes rapid metabolism by abundantly available plasma and tissue esterases, has organ independent metabolism, and a first-order pharmacokinetic profile independent of body weight and elimination clearance, while maintaining a similar safety profile to that of midazolam. ${ }^{7,34}$

Phase II/III general anesthesia trials in Japan have demonstrated the action of remimazolam as a general anesthetic to be "non-inferior" to propofol. It is important to note that remimazolam was found to be associated with longer times to loss of consciousness and time to extubation; however, a few seconds is deemed clinically insignificant, and the authors of the study argued that because remimazolam can be effectively reversed with flumazenil, this benefit outweighs the minor cost. Additionally, extubation time is presumed to be reduced with the implementation of a tapering regimen for remimazolam. ${ }^{7}$

Trials have also demonstrated remimazolam's success over midazolam. In a cohort of 461 colonoscopy patients with randomization to either remimazolam, placebo, or open-label midazolam groups, the rates of successful colonoscopies without rescue medication were 91.3, 1.7, and 25.2, respectively. Furthermore, patients that received remimazolam experienced less hypotension and a faster recovery time than their midazolam counterparts. ${ }^{35}$ In a co- hort of 446 patients that underwent flexible bronchoscopy, procedural success with the group receiving remimazolam was $80.6 \%$, compared to $32.9 \%$ and $4.8 \%$ for midazolam and placebo, respectively. An added benefit of remimazolam here was a shorter time to recovery and a shorter time to alertness and restoration of neuropsychiatric function. ${ }^{36}$

Remimazolam provides a similar degree of sedation with similar patient satisfaction and earlier "readiness to discharge" times. ${ }^{36}$ Although unlikely to replace propofol in patients undergoing colonoscopy, endoscopists will be able to perform the majority of these procedures with remimazolam alone while spared the fear of delayed discharge. Remimazolam will also be a valuable addition to propofol in advanced endoscopic procedures as it does not delay discharge. ${ }^{1}$

\section{PHARMACOKINETICS}

Remimazolam is generally administered IV, as it has negligible oral bioavailability. It is completely absorbed via the GI tract but then undergoes widespread first-pass metabolism. ${ }^{37}$ As previously discussed, remimazolam metabolism is not organ-dependent. Therefore, we may not see the same decline in the inability to metabolize this drug with age as we do with drugs dependent on hepato-renal clearance. ${ }^{1}$ Intranasal sedation using remimazolam has also been studied and determined to be a viable alternative for procedural sedation. It may especially have value for pediatric procedures, where IV sedation can prove difficult. ${ }^{38}$

Remimazolam's onset of action was demonstrated to be 1-3 minutes, surpassing the rapidity of midazolam (3-5 minutes). ${ }^{39}$ This is clinically significant, as it will ease the administration of accurate maintenance doses. ${ }^{40}$ Additionally, the half time of remimazolam is 7-8 minutes, much less than midazolam. ${ }^{1}$

The duration of action remimazolam is eliminated by first-order kinetics at clinical doses. Its volume of distribution is less than that of midazolam. This makes way for rapid elimination without significant accumulation. ${ }^{40} \mathrm{Ad}-$ ditionally, drug clearance seems to be independent of body weight, suggesting that patients can be administered fixed doses of remimazolam. ${ }^{40}$

\section{PHARMACODYNAMICS}

The Modified Observer's Assessment of Alertness/Sedation Scale (MOAA/S) is frequently used to determine a subject's level of sedation in anesthesia drug studies. It is scored $0-5$, where 5 represents an awake and alert subject, and 0 represents a patient under general anesthesia. A study was conducted with continuous infusion of remimazolam, and subjects were assigned scores based on levels of sedation achieved. These scores were then applied to a sigmoid model to better predict the probability of achieving a specific level of sedation for a given concentration of the drug. It was found that there was greater difficulty in achieving moderate levels of sedation (scores 2, 3), and the infusion quickly brought patients from 5 to 0.28

Benzodiazepines are often administered with the intent of strengthening the effect of opioid analgesia. The coad- 
ministration of inhaled remimazolam with inhaled remifentanil has been investigated in rodents, and it was found that remimazolam has the power to potentiate remifentanil's analgesic properties. Additionally, it has been speculated that remifentanil may work to increase the sedative properties of remimazolam. ${ }^{41}$

\section{HEMODYNAMICS}

In one study, blood pressure and heart rate were monitored during continuous administration of IV remimazolam. Subjects experienced decreases in mean arterial blood pressure $(20 \mathrm{mmHg})$ and increases in heart rate $(20 \mathrm{bpm})$. Additionally, $\mathrm{SpO}_{2}$ levels were monitored during infusion, and it was concluded that there were only modest decreases that could be remedied with supplemental oxygen by nasal cannula. ${ }^{28}$ Another study tested the hemodynamic effects of deep sedation with remimazolam in pigs, sheep, and monkeys. It was found that this drug does not seem to induce an excessive amount of cardiac or respiratory depression. ${ }^{6}$

\section{ECG}

Remimazolam was determined not to prolong the PR interval or the QRS duration on ECG. ${ }^{28}$

\section{REMIMAZOLAM FOR GENERAL ANESTHESIA}

Many studies have been conducted regarding the safety and efficacy of remimazolam use for sedation. One study conducted by Sheng et al. assessed the safety and pharmacokinetic and pharmacodynamic properties of remimazolam following a single ascending dose and continuous infusion in a Chinese population. The study was conducted in a double-blind placebo and midazolam controlled manner with a remimazolam dose of $0.025-0.4 \mathrm{mg} / \mathrm{kg}$. In the study, remimazolam was compared to midazolam and assessed for recovery time, induction dosage, and peak sedation effects. The study concluded that for general anesthesia, an induction dosage of $0.2 \mathrm{mg} / \mathrm{kg} / \mathrm{min}$ of remimazolam and a maintenance dose of $1 \mathrm{mg} / \mathrm{kg} / \mathrm{h}$ could be efficacious. In addition, the study observed that remimazolam demonstrated greater sedation and quicker recovery than midazolam. ${ }^{42}$ Similarly, another study by Antonik et al. conducted a phase I, double-blind, placebo, and active-controlled study to assess remimazolam use compared to midazolam. In the study, remimazolam doses of $0.01-0.35 \mathrm{mg} / \mathrm{kg}$ or placebo were given to 10 participants. The study concluded that rapid onset and dose-dependent sedation for remimazolam was observed after doses of $0.05 \mathrm{mg} / \mathrm{kg}$ and higher. In addition, the researchers observed that $0.075-0.20 \mathrm{mg} / \mathrm{kg}$ doses achieved peak sedation with remimazolam with a median recovery time of 10 minutes with remimazolam doses between $0.10-0.15 \mathrm{mg} / \mathrm{kg}$. In addition, as compared to midazolam, remimazolam provided quicker onset and offset compared to midazolam. ${ }^{27}$ Overall, much of the studies conducted comparing remimazolam with midazolam show quicker onset and offset no adverse events and greater efficacy than midazolam.

Further studies assessing the use of remimazolam as compared to propofol for induction of general anesthesia were conducted. In a study conducted by Doi et al., a singleblind, randomized, parallel-group trial was conducted to assess the efficacy of remimazolam versus propofol for induction and maintenance of general anesthesia. In the study, 375 surgical patients were randomized to receive 6 or 12 $\mathrm{mg} / \mathrm{kg} / \mathrm{h}$ of continuous IV remimazolam versus $2-2.5 \mathrm{mg} / \mathrm{kg}$ of IV propofol followed by $4-10 \mathrm{mg} / \mathrm{kg} / \mathrm{h}$ after the loss of consciousness. The study concluded that remimazolam was not inferior to propofol, given the efficacy of remimazolam in inducing general anesthesia. ${ }^{43}$ Finally, although the use of remimazolam was assessed in ASA I and ASA II patients, less research has been conducted on the use of the sedative for ASA III patients. In another study by Doi et al., 67 surgical patients classified as ASA III were randomized into one group receiving $6 \mathrm{mg} / \mathrm{kg} / \mathrm{h}$ infusion of remimazolam while the other group received $12 \mathrm{mg} / \mathrm{kg} / \mathrm{h}$ infusion for maintenance anesthesia. In conclusion, the study found that both infusion regimens were equally safe and efficacious in ASA III surgical patients and was one of the few studies that assessed the use of the sedative in this subset of patients. ${ }^{44}$

\section{REMIMAZOLAM USE IN COLONOSCOPY, ENDOSCOPY, AND BRONCHOSCOPY}

Further studies assessing the use of remimazolam in patients undergoing gastrointestinal procedures such as colonoscopy, endoscopy, and bronchoscopy were conducted. In one study by Worthington et al., the researchers studied the use of remimazolam in volunteers undergoing colonoscopy. In the study, the first part assessed the use of remimazolam during colonoscopy, while the second part assessed the ability to reverse remimazolam with flumazenil. In the study, adequate sedation for colonoscopy was achieved in 33 of 44 subjects and observed that sedation could be rapidly reversed within 1 minute with flumazenil. In addition, after the procedure, subjects recovered with a median time of $<10$ minutes. Finally, no serious adverse events were reported with the use of the drug. ${ }^{45}$ Another study conducted by Pambianco et al. also assessed the use of remimazolam for procedural sedation, specifically for patients undergoing a colonoscopy. In the randomized, double-blind study, active-controlled study, patients received either a dosage of remimazolam or midazolam for sedation before undergoing a colonoscopy. The study found that adequate sedation was obtained for $>92 \%$ of the remimazolam groups compared with $75 \%$ of the midazolam groups with a p-value of $0.007 .{ }^{34}$ The study concluded that given the high success rate with remimazolam and no serious adverse events, further investigation of remimazolam could be conducted. Similar to the study of the drug in patients receiving a colonoscopy, a similar study was conducted in patients undergoing upper endoscopy. In a similar randomized, double-blind study conducted by Borkett et al., patients scheduled to receive an upper endoscopy were randomized to receive either remimazolam or midazolam. In the study, the researchers observed that the onset of sedation with remimazolam was between 1.5-2.5 minutes compared to 5 minutes for midazolam. In conclusion, the study found that patients receiving an upper endoscopy were successfully sedated with a remimazolam dosage of $0.10-0.20 \mathrm{mg} / \mathrm{kg}$ without any adverse events. ${ }^{46}$ Finally, another study by Chen 
et al. was conducted to assess the efficacy and Safety of remimazolam compared to propofol in patients undergoing colonoscopy. In this blinded, randomized, active-controlled trial, 384 patients were separated into remimazolam and propofol groups. The study concluded that the efficacy of remimazolam was non-inferior to propofol, and emergent adverse events were decreased in the remimazolam group compared to the propofol group. ${ }^{47}$ Similarly, a study conducted by Chen et al. also assessed the use of remimazolam in patients undergoing gastrointestinal endoscopy. In this study, a total of 384 scheduled to receive an endoscopy were randomized assigned to receive remimazolam or propofol. The study concluded that remimazolam allowed for faster recovery after sedation as compared with propofol with lower incidences of adverse events such as hypotension and respiratory depression. ${ }^{48}$ Another study conducted by Pastis et al. observed the efficacy and Safety of remimazolam for sedation during flexible bronchoscopy. In the study, the remimazolam was compared with both placebo and midazolam. The study concluded that in the remimazolam group, the success rate was $80.6 \%$ compared to $32.9 \%$ in the midazolam group. Overall, the remimazolam group had a quicker start time of bronchoscopy, quicker time to full alertness, and superior restoration of neuropsychiatric function with remimazolam. ${ }^{36}$ Overall, in all four studies, remimazolam proved to be a far better option than midazolam for a range of endoscopic procedures, such as colonoscopy, endoscopy, and bronchoscopy.

\section{CONCLUSION}

Remimazolam has a quick onset and quick offset with a good safety profile. It does not have a significant effect on hemodynamics, nor is it known to cause cardiac depression, like propofol. These attributes make it a favorable IV anesthetic for a wide array of procedures. A 2020 study by Doi et al. showed non-inferiority when comparing maintenance of general anesthesia with a remimazolam infusion to the control group receiving a propofol infusion in ASA I and II patients. It is still under clinical trials and maybe quite a ways away from replacing our more well-known IV anesthetics, but it has the potential to provide us more predictability and control over our anesthetic. Remimazolam has organ-independent metabolism as it is metabolized by abundant plasma and non-specific tissue esterases. This makes it advantageous for patients with a history of hepatic and/or renal disease. The medication's addiction profile is very similar to midazolam. Thus there is not an increased risk of addiction with its use. It has many advantages and seemingly few disadvantages. A few of its downsides would be complaints of headaches and somnolence after and involuntary movement during infusions. Involuntary movement can be an obvious issue when immobility is crucial in many surgical procedures. But often, patients undergoing a surgical procedure are given muscle relaxants if no contraindications exist, which can help prevent such involuntary movements. As a recently approved medication, further large clinical trials are still warranted to provide the medical community with a better understanding of its properties. Though it has shown many benefits, it will likely take time before it becomes a commonly used adjunct to other sedatives and analgesics.

Submitted: May 28, 2021 EST, Accepted: June 01, 2021 EST 
Table 1. Remimazolam's Clinical Safety and Efficacy

\begin{tabular}{|c|c|c|c|}
\hline $\begin{array}{l}\text { Author } \\
\text { (Year) }\end{array}$ & $\begin{array}{l}\text { Groups Studied } \\
\text { and Intervention }\end{array}$ & Results and Findings & Conclusions \\
\hline $\begin{array}{l}\text { Sheng et al. } \\
\text { (2019) }\end{array}$ & $\begin{array}{l}\text { Healthy Chinese } \\
\text { patients }\end{array}$ & $\begin{array}{l}\text { Sedation function was observed at the dose } \\
\text { of } 0.05 \mathrm{mg} / \mathrm{kg} \text { remimazolam. Doses of } \\
>0.075 \text { exerted a peak sedation effect within } \\
1-2 \mathrm{~min} \text { after injection. For general } \\
\text { anesthesia, an induction dosage of } 0.2 \mathrm{mg} / \\
\mathrm{kg} / \mathrm{min} \text { and a maintenance dosage of } 1 \mathrm{mg} / \\
\mathrm{kg} / \mathrm{h} \text { achieved satisfactory efficacy effect. }\end{array}$ & $\begin{array}{l}\text { Remimazolam was safe and well- } \\
\text { tolerated in healthy Chinese } \\
\text { participants. }\end{array}$ \\
\hline $\begin{array}{l}\text { Antonik et } \\
\text { al. (2012) }\end{array}$ & $\begin{array}{l}\text { Ten cohorts of } \\
\text { health subjects } \\
\text { randomized to } \\
\text { receive a 1-minute } \\
\text { IV infusion of } \\
\text { remimazolam, } \\
\text { midazolam, or } \\
\text { placebo }\end{array}$ & $\begin{array}{l}\text { Pharmacokinetic behavior of remimazolam } \\
\text { linear and its systemic clearance was } \\
\text { approximately three times of midazolam. } \\
\text { Administration of } 0.05 \mathrm{mg} / \mathrm{kg} \text { and higher } \\
\text { doses of remimazolam achieved a rapid } \\
\text { onset and dose-dependent sedation. }\end{array}$ & $\begin{array}{l}\text { Remimazolam provided rapid onset } \\
\text { and offset sedation with minimal } \\
\text { adverse events. Further studies on } \\
\text { the use of remimazolam for } \\
\text { sedation can be conducted. }\end{array}$ \\
\hline $\begin{array}{l}\text { Doi et al. } \\
(2020)\end{array}$ & $\begin{array}{l}\text { Surgical patients } \\
\text { randomized to } \\
\text { remimazolam at } 6 \\
\text { or } 12 \mathrm{mg} / \mathrm{kg} / \mathrm{h} \\
\text { infusion }\end{array}$ & $\begin{array}{l}\text { Remimazolam had a longer time to LOC and } \\
\text { longer time to extubate but overall was non- } \\
\text { inferior compared to propofol }\end{array}$ & $\begin{array}{l}\text { Remimazolam was well tolerated } \\
\text { and non-inferior to propofol for } \\
\text { general anesthesia }\end{array}$ \\
\hline $\begin{array}{l}\text { Worthington } \\
\text { et al. (2013) }\end{array}$ & $\begin{array}{l}\text { Healthy volunteers } \\
\text { receiving } \\
\text { remimazolam for } \\
\text { sedation during } \\
\text { colonoscopy }\end{array}$ & $\begin{array}{l}\text { Over } 70 \% \text { of subjects had successful } \\
\text { sedation with remimazolam with a median } \\
\text { recovery time of }<10 \text { minutes after the } \\
\text { procedure }\end{array}$ & $\begin{array}{l}\text { Remimazolam has success rates } \\
\text { comparable to other drugs for } \\
\text { induction during colonoscopy. } \\
\text { Further studies with the use of } \\
\text { remimazolam in patients receiving } \\
\text { colonoscopy or other Gl } \\
\text { procedures can be conducted }\end{array}$ \\
\hline $\begin{array}{l}\text { Pambianco } \\
\text { et al. (2015) }\end{array}$ & $\begin{array}{l}162 \text { patients aged } \\
18-70 \text { scheduled to } \\
\text { receive a routine } \\
\text { colonoscopy }\end{array}$ & $\begin{array}{l}\text { Single-dose of remimazolam provided }>92 \% \\
\text { success rate for the remimazolam group } \\
\text { compared to } 75 \% \text { for the midazolam group }\end{array}$ & $\begin{array}{l}\text { Remimazolam provided high levels } \\
\text { of success rate for induction during } \\
\text { colonoscopy compared to } \\
\text { midazolam. Further studies } \\
\text { assessing the use of remimazolam } \\
\text { for patients undergoing } \\
\text { colonoscopy or other Gl } \\
\text { procedures can be conducted }\end{array}$ \\
\hline $\begin{array}{l}\text { Borkett et al. } \\
\text { (2015) }\end{array}$ & $\begin{array}{l}\text { Patients scheduled } \\
\text { to undergo a } \\
\text { diagnostic upper } \\
\text { gastrointestinal } \\
\text { endoscopy } \\
\text { randomized to } \\
\text { receive } 1 \text { of } 3 \\
\text { doses of } \\
\text { remimazolam or } \\
\text { midazolam }\end{array}$ & $\begin{array}{l}\text { The onset of sedation in remimazolam dose } \\
\text { group was } 1.5 \text { - } 2.5 \text { minutes compared to } 5 \\
\text { minutes for the midazolam group }\end{array}$ & $\begin{array}{l}\text { A single administration of } \\
\text { remimazolam with a dosage of } \\
0.10-0.20 \mathrm{mg} / \mathrm{kg} \text { was able to induce } \\
\text { patients undergoing diagnostic } \\
\text { upper gastrointestinal endoscopy } \\
\text { with a similar safety profile as } \\
\text { midazolam }\end{array}$ \\
\hline $\begin{array}{l}\text { Chen et al. } \\
(2020)\end{array}$ & $\begin{array}{l}384 \text { patients } \\
\text { undergoing } \\
\text { colonoscopy } \\
\text { randomized to } \\
\text { receive } \\
\text { remimazolam or } \\
\text { propofol }\end{array}$ & $\begin{array}{l}\text { Remimazolam was noted to be non-inferior } \\
\text { to propofol with similar success rates and } \\
\text { induction time of sedation }\end{array}$ & $\begin{array}{l}\text { Remimazolam is non-inferior in } \\
\text { sedation efficacy while safer than } \\
\text { propofol in patients undergoing } \\
\text { colonoscopy }\end{array}$ \\
\hline $\begin{array}{l}\text { Chen et al. } \\
(2020)\end{array}$ & $\begin{array}{l}384 \text { patients } \\
\text { undergoing upper } \\
\text { endoscopy } \\
\text { randomized to } \\
\text { receive } \\
\text { remimazolam or } \\
\text { propofol }\end{array}$ & $\begin{array}{l}\text { Remimazolam was noted to be non-inferior } \\
\text { than propofol, with a shorter time to be fully } \\
\text { alert and lower incidences of hypotension } \\
\text { and respiratory depression }\end{array}$ & $\begin{array}{l}\text { Remimazolam is non-inferior to } \\
\text { propofol and allows for faster } \\
\text { recovery from sedation. In addition, } \\
\text { it appears to be superior to } \\
\text { propofol with adverse events }\end{array}$ \\
\hline $\begin{array}{l}\text { Pastis et al. } \\
\text { (2018) }\end{array}$ & $\begin{array}{l}\text { Patients } \\
\text { undergoing flexible }\end{array}$ & $\begin{array}{l}\text { The success rate in the remimazolam group } \\
\text { was } 80.6 \% \text { compared to } 32.9 \% \text { in the }\end{array}$ & $\begin{array}{l}\text { Remimazolam was effective and } \\
\text { safe for moderate sedation during }\end{array}$ \\
\hline
\end{tabular}




\begin{tabular}{|l|l|l|l|}
\hline & $\begin{array}{l}\text { bronchoscopy } \\
\text { receiving either } \\
\text { remimazolam or } \\
\text { midazolam }\end{array}$ & $\begin{array}{l}\text { midazolam group. In addition, time to full } \\
\text { alertness in the remimazolam group was } \\
\text { shorter compared to midazolam. }\end{array}$ & $\begin{array}{l}\text { bronchoscopy and demonstrated a } \\
\text { shorter onset of action and faster } \\
\text { neuropsychiatric recovery than } \\
\text { midazolam. }\end{array}$ \\
\hline
\end{tabular}




\section{REFERENCES}

1. Goudra BG, Singh PM. Remimazolam: The future of its sedative potential. Saudi Journal of Anaesthesia. 2014;8(3):388-391. doi:10.4103/1658-354X.136627

2. Cornett EM, Novitch MB, Brunk AJ, et al. New benzodiazepines for sedation. Best Practice and Research: Clinical Anaesthesiology. 2018;32(2):149-164. doi:10.1016/j.bpa.2018.06.007

3. Raeder J. Procedural sedation in ambulatory anaesthesia. Current Opinion in Anaesthesiology. 2019;32(6):743-748. doi:10.1097/ACO.0000000000000 $\underline{792}$

4. Sear JW. Challenges of bringing a new sedative to market! Current Opinion in Anaesthesiology. 2018;31(4):423-430. doi:10.1097/ACO.0000000000000 $\underline{614}$

5. Wysowski DK, Pollock ML. Reports of death with use of Propofol (Diprivan) for nonprocedural (longterm) sedation and literature review. Anesthesiology. 2006;105(5):1047-1051. doi:10.1097/00000542-20061 1000-00027

6. Pambianco DJ, Cash BD. New horizons for sedation: The ultrashort acting benzodiazepine remimazolam. Published online 2016. doi:10.1016/j.tgie.2016.02.004

7. Wesolowski AM, Zaccagnino MP, Malapero RJ, Kaye AD, Urman RD. Remimazolam: Pharmacologic Considerations and Clinical Role in Anesthesiology. Pharmacotherapy: The Journal of Human Pharmacology and Drug Therapy. 2016;36(9):1021-1027. doi:10.100 2/phar.1806

8. Griffin CE, Kaye AM, Rivera Bueno F, Kaye AD. Benzodiazepine pharmacology and central nervous system-mediated effects. Ochsner Journal. 2013;13(2):214-223.

9. Pergolizzi J, Böger RH, Budd K, et al. Opioids and the management of chronic severe pain in the elderly: Consensus statement of an international expert panel with focus on the six clinically most often used world health organization step III opioids (Buprenorphine, Fentanyl, Hydromorphone, Met. Pain Practice. 2008;8(4):287-313. doi:10.1111/j.1533-2500.2008.002 $\underline{04 . X}$

10. Kelly MD, Smith A, Banks G, et al. Role of the histidine residue at position 105 in the human $\alpha 5$ containing $\mathrm{GABA}_{\mathrm{A}}$ receptor on the affinity and efficacy of benzodiazepine site ligands. British Journal of Pharmacology. 2002;135(1):248-256. doi:10.1038/s j.bjp.0704459
11. Kanto J, Klotz U. Intravenous Benzodiazepines as Anaesthetic Agents: Pharmacokinetics and Clinical Consequences. Acta Anaesthesiologica Scandinavica. 1982;26(6):554-569. doi:10.1111/j.1399-6576.1982.tb 01817.x

12. Calcaterra NE, Barrow JC. Classics in chemical neuroscience: Diazepam (valium). ACS Chemical Neuroscience. 2014;5(4):253-260. doi:10.1021/cn5000 $\underline{056}$

13. PRESKORN SH. A Way of Conceptualizing Benzodiazepines to Guide Clinical Use. Journal of Psychiatric Practice. 2015;21(6):436-441. doi:10.1097/ PRA.0000000000000114

14. Becker DE. Pharmacodynamic considerations for moderate and deep sedation. Anesthesia progress. 2012;59(1):28-42. doi:10.2344/0003-3006-59.1.28

15. Haefely W. Benzodiazepine interactions with GABA receptors. Neuroscience Letters. 1984;47(3):201-206. doi:10.1016/0304-3940(84)9051 4-7

16. McLean MJ, Macdonald RL. Benzodiazepines, but not beta-carbolines, limit high frequency repetitive firing of action potentials of spinal cord neurons in cell culture. Journal of Pharmacology and Experimental Therapeutics. 1988;244(2).

17. Krieman MJ, Hershock DM, Greenberg IJ, Vogel WH. Effects of adinazolam on plasma catecholamine, heart rate and blood pressure responses in stressed and non-stressed rats. Neuropharmacology. 1992;31(1):33-38. doi:10.1016/0028-3908(92)90157-K

18. Paradis S, Leoni V, Caccia C, Berdeaux A, Morin D. Cardioprotection by the TSPO ligand $4^{\prime}$ chlorodiazepam is associated with inhibition of mitochondrial accumulation of cholesterol at reperfusion. Cardiovascular Research. 2013;98(3):420-427. doi:10.1093/cvr/cvt079

19. Yousefi OS, Wilhelm T, Maschke-Neuß K, et al. The 1,4-benzodiazepine Ro5-4864 (4-chlorodiazepam) suppresses multiple proinflammatory mast cell effector functions. Cell Communication and Signaling. 2013;11(1). doi:10.118 6/1478-811X-11-13

20. Matsumoto T, Ogata M, Koga K, Shigematsu A. Effect of peripheral benzodiazepine receptor ligands on lipopolysaccharide-induced tumor necrosis factor activity in thioglycolate- treated mice. Antimicrobial Agents and Chemotherapy. 1994;38(4):812-816. doi:1 0.1128/AAC.38.4.812 
21. Buchwald P, Bodor N. Recent advances in the design and development of soft drugs. Pharmazie. Published online 2014. doi:10.1691/ph.2014.3911R

22. Sneyd JR. Remimazolam: New beginnings or just a me-too? Anesthesia and Analgesia. Published online 2012. doi:10.1213/ANE.0b013e31823acb95

23. Kilpatrick GJ, McIntyre MS, Cox RF, et al. CNS 7056: A novel ultra-short-acting benzodiazepine. Anesthesiology. Published online 2007. doi:10.1097/0 1.anes.0000267503.85085.c0

24. Zhou Y, Hu P, Jiang J. Metabolite characterization of a novel sedative drug, remimazolam in human plasma and urine using ultra-high-performance liquid chromatography coupled with synapt highdefinition mass spectrometry. Journal of Pharmaceutical and Biomedical Analysis. Published online 2017. doi:10.1016/j.jpba.2017.01.016

25. Birgenheier NM, Stuart AR, Egan TD. Soft drugs in anesthesia: remifentanil as prototype to modern anesthetic drug development. Current opinion in anaesthesiology. Published online 2020. doi:10.1097/A $\underline{\mathrm{C} 0.0000000000000879}$

26. Schippers F, Pesic M, Saunders R, et al. Randomized Crossover Trial to Compare Abuse Liability of Intravenous Remimazolam Versus Intravenous Midazolam and Placebo in Recreational Central Nervous System Depressant Users. Journal of Clinical Pharmacology. Published online 2020. doi:1 0.1002/jcph.1614

27. Antonik LJ, Goldwater DR, Kilpatrick GJ, Tilbrook GS, Borkett KM. A Placebo- and MidazolamControlled Phase I Single Ascending-Dose Study Evaluating the Safety, Pharmacokinetics, and Pharmacodynamics of Remimazolam (CNS 7056). Anesthesia \& Analgesia. 2012;115(2):274-283. doi:1 $\underline{0.1213 / A N E .0 b 013 e 31823 f 0 c 28}$

28. Schüttler J, Eisenried A, Lerch M, Fechner J, Jeleazcov C, Ihmsen H. Pharmacokinetics and pharmacodynamics of remimazolam (CNS 7056) after continuous infusion in healthy male volunteers Part I. Pharmacokinetics and clinical pharmacodynamics. Anesthesiology. Published online 2020. doi:10.1097/A LN.0000000000003103

29. Goudra B, Singh PM, Lichtenstein GR. Medical, Political, and Economic Considerations for the Use of MAC for Endoscopic Sedation: Big Price, Little Justification? Digestive Diseases and Sciences. 2020;65(9):2466-2472. doi:10.1007/s10620-020-0646 $\underline{4-3}$

30. Goudra B, Singh P. Propofol alternatives in gastrointestinal endoscopy anesthesia. Saudi Journal of Anaesthesia. 2014;8(4):540. doi:10.4103/1658-354 X.140893
31. Riphaus A, Macias-Gomez C, Devière J, Dumonceau JM. Propofol, the preferred sedation for screening colonoscopy, is underused. Results of an international survey. Digestive and Liver Disease. 2012;44(5):389-392. doi:10.1016/j.dld.2011.10.019

32. Urits I, Viswanath O, Orhurhu V, et al. The Utilization of Mu-Opioid Receptor Biased Agonists: Oliceridine, an Opioid Analgesic with Reduced Adverse Effects. Current Pain and Headache Reports. 2019;23(5). doi:10.1007/s11916-019-0773-1

33. Goudra B, Singh P. Oliceridine and its potential to revolutionize GI endoscopy sedation. Saudi Journal of Anaesthesia. 2020;14(3):349-354. doi:10.4103/sja.SI A $813 \quad 19$

34. Pambianco DJ, Borkett KM, Riff DS, et al. A phase IIb study comparing the safety and efficacy of remimazolam and midazolam in patients undergoing colonoscopy. Gastrointestinal Endoscopy. 2016;83(5):984-992. doi:10.1016/j.gie.2015.08.062

35. Rex DK, Bhandari R, Desta T, et al. A phase III study evaluating the efficacy and safety of remimazolam (CNS 7056) compared with placebo and midazolam in patients undergoing colonoscopy. Gastrointestinal Endoscopy. 2018;88(3):427-437.e6. do i:10.1016/i.gie.2018.04.2351

36. Pastis NJ, Yarmus LB, Schippers F, et al. Safety and Efficacy of Remimazolam Compared With Placebo and Midazolam for Moderate Sedation During Bronchoscopy. Chest. 2019;155(1):137-146. doi:10.10 16/j.chest.2018.09.015

37. Pesic M, Stöhr T, Ossig J, et al. Remimazolam Has Low Oral Bioavailability and No Potential for Misuse in Drug-Facilitated Sexual Assaults, with or Without Alcohol: Results from Two Randomised Clinical Trials. Drugs in R and D. Published online 2020. doi:1 $\underline{0.1007 / \mathrm{s} 40268-020-00317-0}$

38. Pesic M, Schippers F, Saunders R, Webster L, Donsbach M, Stoehr T. Pharmacokinetics and pharmacodynamics of intranasal remimazolam-a randomized controlled clinical trial. European Journal of Clinical Pharmacology. Published online 2020. doi:1 0.1007/s00228-020-02984-Z

39. Wesolowski AM, Zaccagnino MP, Malapero RJ, Kaye AD, Urman RD. Remimazolam: Pharmacologic Considerations and Clinical Role in Anesthesiology. Pharmacotherapy: The Journal of Human Pharmacology and Drug Therapy. 2016;36(9):1021-1027. doi:10.100 2/phar.1806 
40. Wiltshire HR, Kilpatrick GJ, Tilbrook GS, Borkett $\mathrm{KM}$. A placebo-and midazolam-controlled phase i single ascending-dose study evaluating the safety, pharmacokinetics, and pharmacodynamics of remimazolam (CNS 7056): Part II. population pharmacokinetic and pharmacodynamic modeling and simulation. Anesthesia and Analgesia. Published online 2012. doi:10.1213/ANE.0b013e318241f68a

41. Bevans T, Deering-Rice C, Stockmann C, Rower J, Sakata D, Reilly C. Inhaled remimazolam potentiates inhaled remifentanil in rodents. Anesthesia and Analgesia. Published online 2017. doi:10.1213/ANE.00 $\underline{00000000002022}$

42. Sheng X yan, Liang Y, Yang X yuan, et al. Safety, pharmacokinetic and pharmacodynamic properties of single ascending dose and continuous infusion of remimazolam besylate in healthy Chinese volunteers. European Journal of Clinical Pharmacology. 2020;76(3):383-391. doi:10.1007/s00228-019-02800-3

43. Doi M, Morita K, Takeda J, Sakamoto A, Yamakage M, Suzuki T. Efficacy and safety of remimazolam versus propofol for general anesthesia: a multicenter, single-blind, randomized, parallel-group, phase IIb/ III trial. Journal of Anesthesia. 2020;34(4):543-553. do i:10.1007/s00540-020-02788-6

44. Doi M, Hirata N, Suzuki T, Morisaki H, Morimatsu $\mathrm{H}$, Sakamoto A. Safety and efficacy of remimazolam in induction and maintenance of general anesthesia in high-risk surgical patients (ASA Class III): results of a multicenter, randomized, double-blind, parallelgroup comparative trial. Journal of Anesthesia. 2020;34(4):491-501. doi:10.1007/s00540-020-0277 $6-\mathrm{w}$
45. Worthington MT, Antonik LJ, Goldwater DR, et al. A phase ib, dose-finding study of multiple doses of remimazolam (CNS 7056) in volunteers undergoing colonoscopy. Anesthesia and Analgesia.

2013;117(5):1093-1100. doi:10.1213/ANE.0b013e3182 a705ae

46. Borkett KMC, Riff DS, Schwartz HI, et al. A Phase IIa, Randomized, Double-Blind Study of Remimazolam (CNS 7056) Versus Midazolam for Sedation in Upper Gastrointestinal Endoscopy. Anesthesia and Analgesia. 2015;120(4):771-780. doi:1 $\underline{0.1213 / A N E .0000000000000548}$

47. Chen S, Wang J, Xu X, et al. The efficacy and safety of remimazolam tosylate versus propofol in patients undergoing colonoscopy: a multicentered, randomized, positive-controlled, phase III clinical trial. American journal of translational research. 2020;12(8):4594-4603.

48. Chen SH, Yuan TM, Zhang J, et al. Remimazolam tosilate in upper gastrointestinal endoscopy: A multicenter, randomized, non-inferiority, phase III trial. Journal of Gastroenterology and Hepatology (Australia). Published online 2020. doi:10.1111/igh.15 $\underline{188}$ 